\title{
Identification of Genes with Altered Methylation and Its Role in Early Diagnosis of Sepsis-Induced Acute Respiratory Distress Syndrome
}

This article was published in the following Dove Press journal: International Journal of General Medicine

\author{
Jihua Feng* \\ Jielong Pang* \\ Dan $\mathrm{He}^{*}$ \\ Zimeng Wu \\ Qian Li \\ Pan Ji \\ Cuiying $\mathrm{He}$ \\ Zhimei Zhong \\ Hongyuan Li \\ Jianfeng Zhang (D)
}

Department of Emergency Medicine, The Second Affiliated Hospital of Guangxi Medical University, Nanning 530007,

People's Republic of China

*These authors contributed equally to this work
Correspondence: Jianfeng Zhang

Department of Emergency Medicine, The Second Affiliated Hospital of Guangxi

Medical University, Nanning 530007,

People's Republic of China

Email drzhangjf@I63.com
Purpose: Early diagnosis of sepsis-induced acute respiratory distress syndrome (ARDS) is critical for effective treatment. We aimed to identify early stage biomarkers.

Materials and Methods: Differentially expressed genes were identified in whole blood samples from patients with sepsis or ARDS based on the Gene Expression Omnibus (GEO) datasets GSE32707, GSE54514 and GSE10361. Functional enrichment analysis explored the biological characteristics of differentially expressed genes. Genes with high functional connectivity based on a protein-protein interaction network were marked as hub genes, which were validated using the GEO dataset GSE76293, and a gene set variation analysis index (GSVA) was assigned. Diagnostic and predictive ability of the hub genes were assessed by receiver operating characteristic (ROC) curve analysis. DNA methylation levels of hub genes were quantified using the GEO dataset GSE67530.

Results: Forty-one differentially expressed genes were shared between sepsis-specific and ARDS-specific datasets. MAP2K2 and IRF7 functional activity was highly connected in sepsis-induced ARDS. Hub genes included RETN, MVP, DEFA4, CTSG, AZU1, FMNL1, RBBP7, POLD4, RIN3, IRF7. ROC curve analysis of the hub gene GSVA index showed good diagnostic ability in sepsis or ARDS. Among genes related to sepsis-induced ARDS, 17 were differentially methylated. Principal component analysis and heatmaps indicated that gene methylation patterns differed significantly between ARDS patients and controls.

Conclusion: We identified a genetic profile specific to early-stage sepsis-induced ARDS. The abnormal expression of these genes may be caused by hypomethylation, which may serve as a biomarker for early diagnosis of ARDS.

Keywords: acute respiratory distress syndrome, ARDS, sepsis-induced ARDS, hub genes, methylation, gene set variation analysis

\section{Introduction}

Acute respiratory distress syndrome (ARDS) is associated with a variety of pathological changes, including the release of inflammatory cytokines, the destruction of pulmonary vascular endothelial cells, the loss of surfactant, the accumulation of lung fluid and the formation of excessive fibrosis. ${ }^{1}$ Sepsis is a common risk factor for ARDS and patient prognosis is worsened when sepsis is present. ${ }^{2}$ The 60 -day mortality rate of patients with sepsis-related ARDS is significantly higher than that of patients with ARDS unrelated to sepsis. Sepsis-induced ARDS develops rapidly, which limits the time available for doctors to diagnose and treat patients. ${ }^{2}$ However, few predictive markers of sepsis-induced ARDS are known. 
Previous work has addressed sepsis-induced ARDS risk prediction by focusing on the transcriptome and clinical indicators. ${ }^{3,4}$ Although some biomarkers have been confirmed, most have been correlated with prognosis rather than early diagnosis. A previous study demonstrated that Interferon (INF) response factors IRF7 signaling regulated pulmonary inflammatory response through the TLR4 signaling pathway. ${ }^{5}$ Activating transcription factor 4 (ATF4) regulates genes involved in the inflammatory response. $^{6}$ AUZ1 in the inflammatory process is known to facilitate leukocyte-mediated migration, and neutrophil activation and degranulation process. ${ }^{7}$ In contrast, tissue factor was identified as not only a diagnostic biomarker of sepsis-induced ARDS, but also a prognostic indicator of ARDS. $^{2}$ Another study elucidated the diagnostic role of 11 biomarkers in ARDS, but did not distinguish between the biomarkers of traumatic or non-traumatic ARDS. ${ }^{8}$

Epigenetic changes, especially DNA methylation, are critical regulators of gene transcription, and therefore influence the occurrence and development of diseases. ${ }^{9}$ Changes in DNA methylation have been implicated in many diseases, including lung cancer and airway inflammation. ${ }^{10,11}$ DNA methylation can directly block transcription by inhibiting the binding of specific transcription factors to target sequences in genes. ${ }^{12-14}$ For example, sepsis-induced ARDS in animal models can be induced using lipopolysaccharide, which alters DNA methylation to up-regulate the expression of certain inflammatory factors. ${ }^{15}$ Lipopolysaccharide induces methylation not only of tumor necrosis factor-alpha promoter in human THP-1 monocytes, but also of sequences in the HIC-1 gene in mouse embryonic fibroblasts lacking p53. ${ }^{16}$ In human intestinal epithelial cells, lipopolysaccharide stimulates the methylation of regions in the IL-8 promoter with which the histone residues H3K4, H3K9 and H3K27 interact. ${ }^{17}$ Since sepsis-induced ARDS is closely related to the level of lipopolysaccharide in the blood stream, aberrant DNA methylation in lung cells could be considered as a prophase event in sepsis-induced ARDS. However, the methylation profile of genes associated with ARDS or sepsis has not been fully studied. One study showed that epigenetic variation of myosin light chain kinase is involved in the pathogenesis of ARDS and might influence risk of mortality among patients. ${ }^{18}$ However, that study focused only on a single genetic variation, which may not be diagnostic for all patients.

In this in silico study, we comprehensively analyzed differences in gene expression and methylation among patients with sepsis alone, patients with sepsis-related ARDS and healthy controls. The results may be helpful for the early diagnosis of ARDS and the development of sepsis treatment strategies.

\section{Materials and Methods \\ Data Preparation}

We extracted information from datasets downloaded from the Gene Expression Omnibus (GEO, https://www.ncbi.nlm. nih.gov/geo/). The GSE32707 gene expression profiles, ${ }^{19}$ obtained using the GPL10558 platform, contained 144 human blood samples, including 58 from patients with sepsis, 31 from patients with sepsis+ARDS, 21 from patients with systemic inflammatory response syndrome and 34 healthy controls. All patients were undergoing mechanical ventilation. The 21 systemic inflammatory response syndrome samples were excluded from our analysis. The GSE10361 gene expression profile data, obtained using the GPL96 platform, comprised 9 human blood samples, including 6 from patients with ARDS and 3 from healthy controls. Among them, 6 samples were collected at $0 \mathrm{~h}$, and 3 at 72 h. The GSE54514 gene expression profile data, obtained with the GPL6947 platform, included 163 human blood samples from up to 5 days for sepsis survivors $(n=26)$, sepsis nonsurvivors $(\mathrm{n}=9)$, and healthy controls $(\mathrm{n}=18)$. Among them, 127 were from patients with sepsis on day_1, day_2, day_3, day_4 and day_5, as well as 36 were from healthy controls on day_1 and day_5. The above three data sets were used to identify hub genes.

The GSE76293 gene expression profile data, ${ }^{20}$ obtained using the platform GPL6883, contained samples of alveolar lavage fluid or blood neutrophils from 12 patients with ARDS and samples of blood neutrophils from 82 healthy controls. All patients in this dataset received ventilation therapy. And the study was split in to 2 parts. PartA: PMN were isolated from ARDS patients alongside PMN from paired (ie, age and gender-matched) HVTs. PartB: PMNs from HVTs cultured with GM-CSF and PI3K Inhibitors. This data set was used to validate the aberrant expression of hub genes. The GSE67530 DNA methylation data, based on the GPL13534 platform, came from blood samples from 39 patients with ARDS, 75 nonARDS ICU controls, and 30 healthy controls.

The normalizeBetweenArrays function in the limma package ${ }^{21}$ was applied to normalize all the gene expression profiles. If a gene was detected using multiple probes, the average expression value for all probes was used as the 
expression value for the gene. All the analyses were performed using R software version 3.5.3. (https://www.r-project.org/). The workflow of the present study is shown in Figure 1.

\section{Differentially Expressed Gene Analysis (DEG)}

The limma package in R was used to screen GSE32707, GSE10361 and GSE54514 datasets to identify genes differentially expressed among sepsis, ARDS and control samples. The fold change (FC) in the expression of individual genes was calculated, and genes with $\left|\log _{2} \mathrm{FC}\right|>1$ and $\mathrm{P}<0.05$ were considered significant. In the GSE32707A dataset, genes related to sepsis-induced ARDS were defined as DEGs that were common to ARDS and sepsis samples (when compared to control), and for which $\mid \log _{2} \mathrm{FC}_{\mathrm{ARDS}}$ vs control $|>| \log _{2}$ $\mathrm{FC}_{\text {sepsis }}$ vs control ${ }^{22}$ The DEGs related to sepsis-induced ARDS that were common to the GSE10361 and GSE54514 datasets were extracted for subsequent analysis.

\section{Identification of Differentially Methylated Genes}

In dataset GSE67530, the mean $\beta$ values of all $\mathrm{CpG}$ islands corresponding to a gene were used to indicate the degree of methylation of that gene. ${ }^{23}$ Differentially methylated genes were identified between ARDS and healthy control samples using the limma package. $\mathrm{P}<0.05$ was considered significant.

\section{Gene Set Variation Analysis (GSVA) and Receiver Operating Characteristic (ROC) Curve Analysis}

GSVA, ${ }^{24}$ as implemented in the GSVA package in R, was used to score individual samples against the DEGs related to sepsis-induced ARDS, and each sample received an upregulated and a down-regulated GSVA score. Samples in the GSE32707, GSE10361 and GSE54514 datasets were scored in this way. The GSVA results were also displayed as a heatmap, and their diagnostic value was assessed using ROC curve analysis with the pROC software package in $\mathrm{R}^{25}$

\section{Functional Enrichment Analysis}

To explore the biological function of the shared upregulated and down-regulated genes in the three gene sets, Gene Ontology (GO) and Kyoto Encyclopedia of Genes and Genomes (KEGG) pathway enrichment analyses were performed using the clusterProfiler package ${ }^{26}$ in $\mathrm{R}$. P value $<0.05$ after adjusting for the false discovery rate was considered significant.

\section{Protein-Protein Interaction (PPI)} Network and Identification of Hub Genes Using the STRING database (https://string-db.org/), ${ }^{27}$ we constructed a PPI network of the shared up-regulated and down-regulated genes from the three gene sets, which was visualized using Cytoscape software. ${ }^{28}$ The KEGG pathways

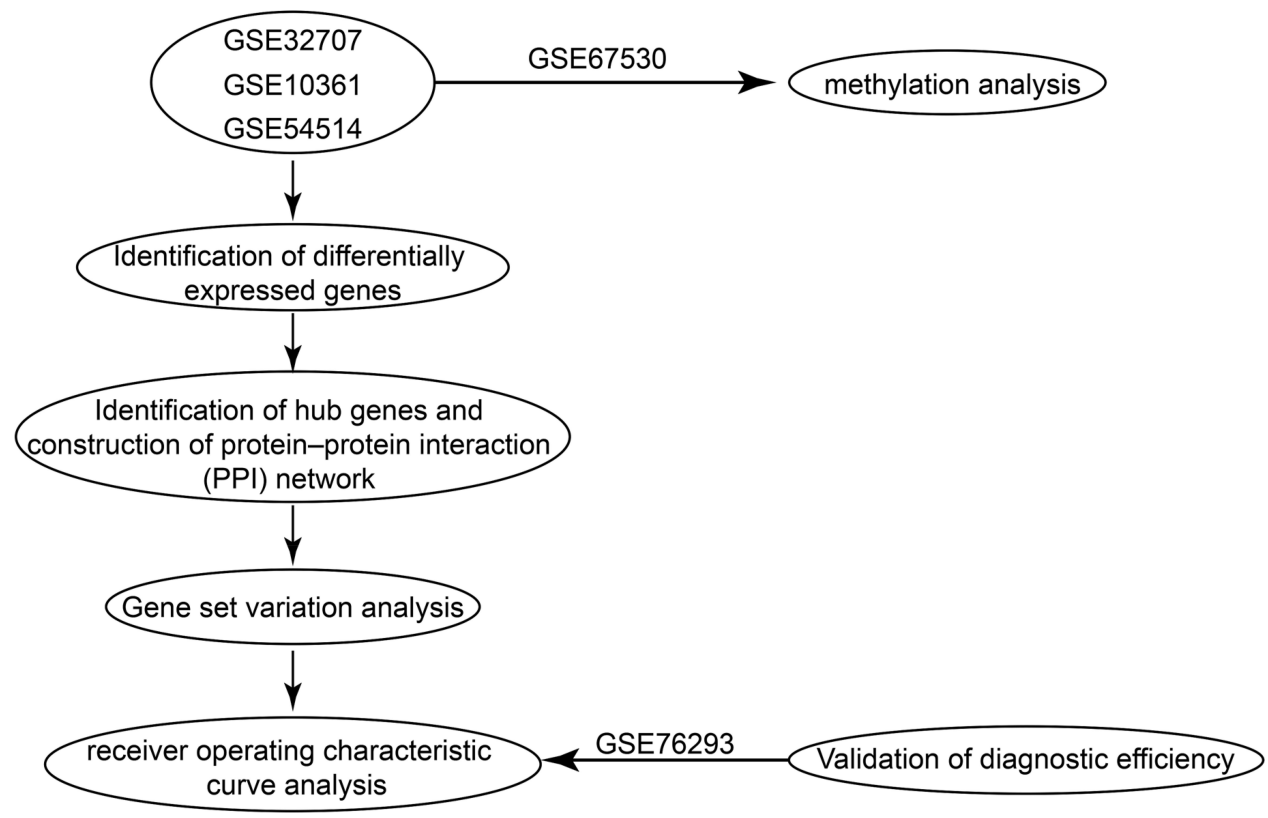

Figure I The workflow of the present study. 
in which these genes were involved, based on functional enrichment analysis, were also displayed in this network. In the PPI network, according to the semantic similarities of GO terms used for gene annotation, we ranked the gene inside the interactome by the average functional similarities between the gene and its interaction partners. Genes with a higher mean functional similarity were considered more crucial. ${ }^{29}$ In addition, we calculated the $\mathrm{Pi}$ value $=\log \mathrm{FC} \times \log (\mathrm{P}$ value $)$ in the three comparisons for the genes based on previous work. $^{30}$ Thus, the weight $(\mathrm{W})$ value was defined as $\mathrm{W}=$ mean functional similarity $\times$ mean $\mathrm{Pi}$. The $\mathrm{W}$ value was used to rank the top 10 genes, which were considered as hub genes. The hub GSVA score was calculated and ROC curve analysis performed as described above.

\section{Results}

\section{Identification of DEGs Involved in Sepsis-Induced ARDS}

In the GSE32707 dataset, 8354 DEGs were obtained between sepsis samples and healthy control samples, and 7859 were obtained between ARDS samples and control samples. A total of 1049 up-regulated genes and 2472 down-regulated genes were shared between sepsis and ARDS samples and were therefore potentially related to sepsis-induced ARDS. In the GSE10361 dataset, there were 1146 up-regulated genes and 969 down-regulated genes in the ARDS samples. In the GSE54514 dataset, there were 2502 up-regulated genes and 2010 downregulated genes in sepsis samples (Figure 2A). The genes potentially related to sepsis-induced ARDS were crossreferenced against the ARDS-related genes and sepsisrelated genes, and the overlapping set of 24 up-regulated and 17 down-regulated genes (Figure 2B) were defined as being related to sepsis-induced ARDS and analyzed further.

\section{Validation of GSVA Scores for Genes Related to Sepsis-Induced ARDS}

ROC curve analysis was performed using the GSVA scores calculated separately for the up-regulated genes related to sepsis-induced ARDS and for the downregulated genes related to sepsis-induced ARDS. Both GSVA scores showed good potential for diagnosing sepsis and ARDS across multiple datasets (Figure 2C). A heatmap was used to correlate the expression of genes related to sepsis-induced ARDS and the two GSVA scores (Figure 2D).

\section{Biological Processes and Pathways Involved in Sepsis-Induced ARDS}

GO analysis of genes related to sepsis-induced ARDS identified 20 biological processes that were significantly enriched, including cellular immune response, neutrophil response to bacteria, neutrophil activation and protein modification (Figure 3A). KEGG pathway enrichment analysis showed a total of 19 pathways, such as the citrate cycle, cellular senescence, toll-like receptor (TLR) signaling and HIF-1 signaling (Figure 3B).

\section{Potential Molecular Mechanism of Hub Genes Driving Development of Sepsis-Induced ARDS}

The 41 genes related to sepsis-induced ARDS were introduced into a PPI network using the STRING database (Figure 4A). We found a high degree of connectivity between $M A P 2 K 2$ and $I R F 7$, which may suggest a bridge role in sepsis-induced ARDS (Figure 4B). During sepsis, lipopolysaccharide activates immune cells by making direct contact with toll-like receptor (TLR)-4. Consequently, a large signaling complex is formed around TLR-4, containing the signal adapter MyD88 and members of the IRAK family that lead to an inflammatory response. ${ }^{31,32}$ Most proteins associated with the TLR signaling pathway are positively regulated during inflammation. MyD88 can mediate the development of lung inflammation induced by mechanical ventilation in mice. $^{33}$ IRAK1 is related to the prognosis of sepsis patients. Up-regulating MAP2K2 and IRF7 may ultimately promote the release of inflammatory factors and activate interferon signaling. Similarity analysis was used to screen for hub genes (Figure 4C). Genes with similar functions were identified as hub genes, including RETN, MVP, DEFA4, CTSG, AZU1, FMNL1, RBBP7, POLD4, RIN3, IRF7. Next we evaluated the biological functions and expression of the identified hub genes in sepsis and ARDS (Figure 4D).

\section{Diagnostic Potential of the GSVA Score for the Set of Hub Genes}

ROC curve analysis of the hub gene GSVA score showed good diagnostic ability in sepsis or ARDS 

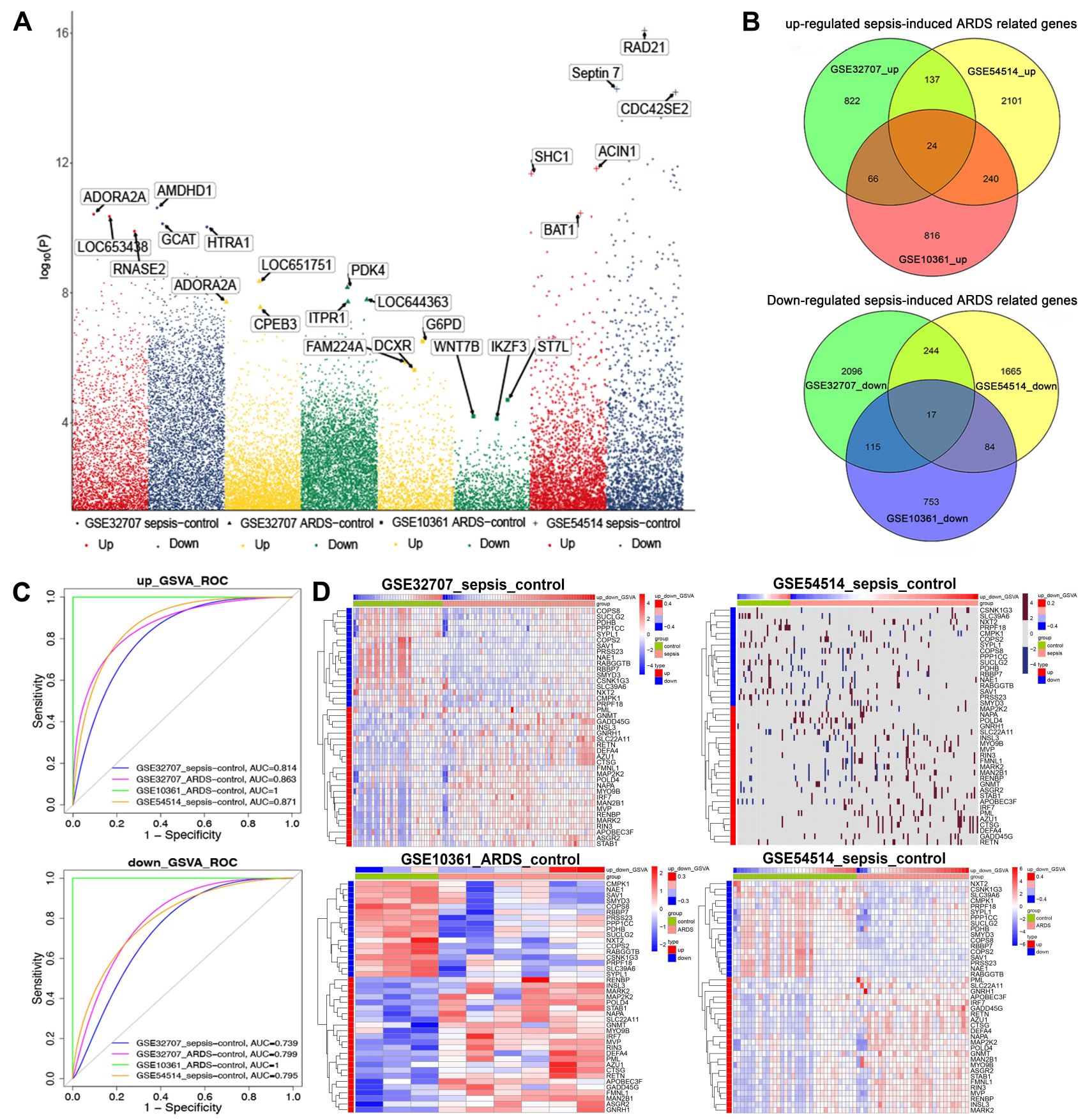

Figure 2 Differentially expressed genes in sepsis-induced ARDS. (A) Manhattan plot of differentially expressed genes. (B) Venn diagram identifying sepsis-specific genes and ARDS-specific genes in different datasets. Up- or down-regulated genes in GSE32707, GSEI036I and GSE545I4 datasets were considered potentially related to sepsisinduced ARDS. (C) Receiver operating characteristic (ROC) curve analysis of potential gene markers based on gene set variation analysis (GSVA) scores. (D) Heatmaps depicting the expression of sepsis-induced ARDS related genes and the corresponding GSVA scores.

(Figure 5A). The expression of hub genes in ARDS or sepsis samples is shown in heatmaps and disease development (Figure 5B and C). Validation in an independent data set (GSE76293) confirmed that the hub gene GSVA score had biomarker potential, with an area under the ROC curve of 0.742 (Figure 5D).

\section{Differential Methylation Alters Hub Gene Expression in Sepsis-Induced ARDS}

A total of 12,557 differentially methylated genes were identified in the GSE67530 set (Figure 6A). Among the 41 genes related to sepsis-induced ARDS, 17 were differentially 

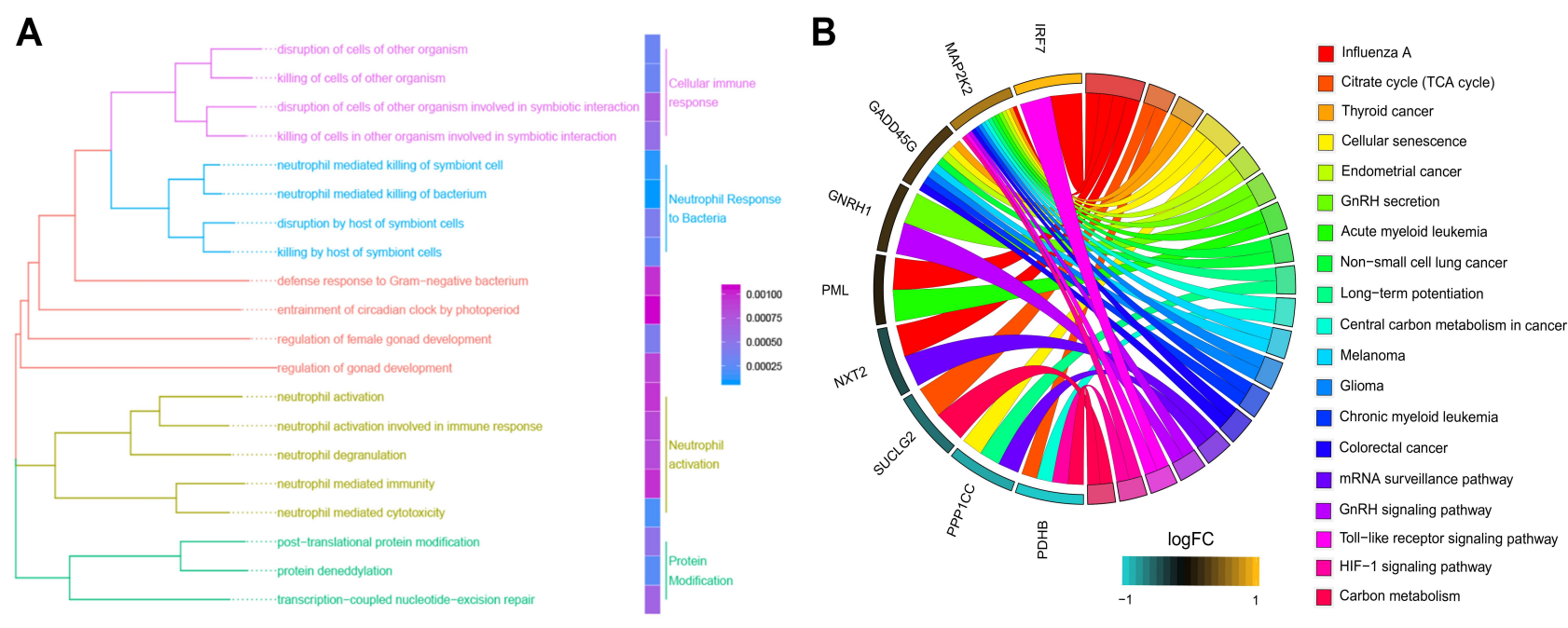

Figure 3 Functional enrichment analysis for the sepsis-induced ARDS related genes. analysis. (B) KEGG analysis showed pathways associated with sepsis-induced ARDS.

methylated. Ten genes showed high expression and low methylation: ASGR2, AZU1, CTSG, DEFA4, GNMT, NAPA, $P M L, R E T N, R I N 3$, and $S T A B 1$. The remaining seven genes showed low expression and high methylation: $C M P K 1$, CSNK1G3, RABGGTB, SAV1, SLC39A6, SUCLG2, SYPL1 (Figure 6B). Gene methylation differed significantly between ARDS and control samples, based on principal component analysis (Figure 6C) and a heatmap (Figure 6D).

\section{Discussion}

Sepsis-induced ARDS is severe and develops rapidly. Although the prognosis of patients with this condition has improved, major treatments have not emerged, in part due to the lack of early diagnostic biomarkers. Therefore, we compared gene expression profiles in patients with sepsis or ARDS in order to understand transcriptional disturbance in sepsis-induced ARDS. We discovered hub genes in sepsis-induced ARDS and uncovered different methylation patterns that may regulate hub gene expression, which may help drive the disease.

The majority of the previous studies mainly focused on the prognosis of ARDS, and few people made further exploration on the early diagnosis of ARDS. We found that in patients with sepsis-induced ARDS, differentially expressed genes were mainly involved in the TCA cycle, cellular senescence, Toll-like receptor signaling pathway and HIF-1 signaling pathway. Studies had confirmed that these pathways were closely related to sepsis and play a key role in the development of sepsis. In particular, toll-like receptor signaling pathway played an important role in the development of sepsis and ARDS. The related research by
Huang et $\mathrm{al}^{34}$ confirmed that anti-TLR $4 \mathrm{mAb}$ could reduce lung injury in mechanical ventilation, inflammation and edema by inhibiting the TLR 4/MyD 88 signaling pathway in rats. Our results also confirmed the above point.

PPI networks were used to unearth functionally related hub genes from three datasets in sepsis-induced ARDS, and these genes were validated using an independent dataset. The hub genes hold great potential as biomarkers as their related proteins are highly expressed in the blood of ARDS patients. Indeed, we confirmed these hub genes show diagnostic potential for sepsis-induced ARDS.

DNA methylation plays an important role in regulating gene transcription. ${ }^{9}$ For example, hypermethylation of $\mathrm{CpG}$ islands leads to gene silencing and disease, which is why demethylation drugs can be effective. ${ }^{35,36}$ Indeed, altered DNA methylation in the lungs has often been connected to tumorigenesis and airway inflammation. ${ }^{10,11}$ However, variation in the methylation of only one gene has so far been linked to ARDS. ${ }^{37,38}$ Our study showed that highly expressed hub genes showed low methylation levels suggestive of hypomethylation. Hypomethylation of the transcriptional start site and first exon of $A Z U 1$ triggers inflammation signaling cascades in multiple autoimmune diseases. ${ }^{39}$ Inflammation may lead to hypomethylation and subsequent up-regulation of DEFA4. ${ }^{40}$ These findings lead us to propose that hypomethylation-induced up-regulation of hub genes contributes to the development of sepsisinduced ARDS. Regrettably, IRF7 was upregulated and highly affecting the toll like receptor signaling pathway. However, IRF7 was not differentially methylated. 
A

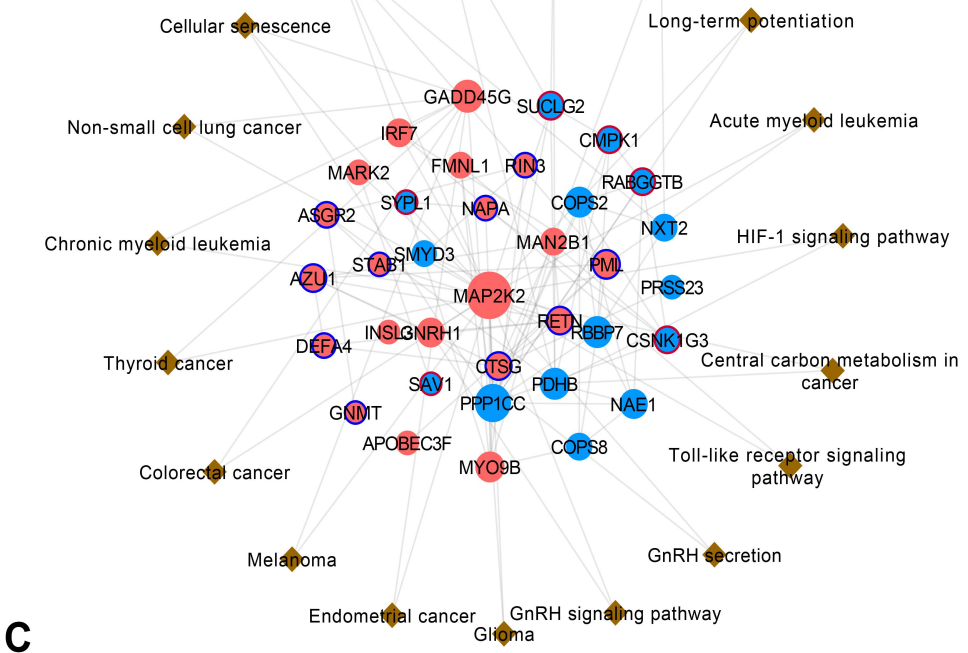

C

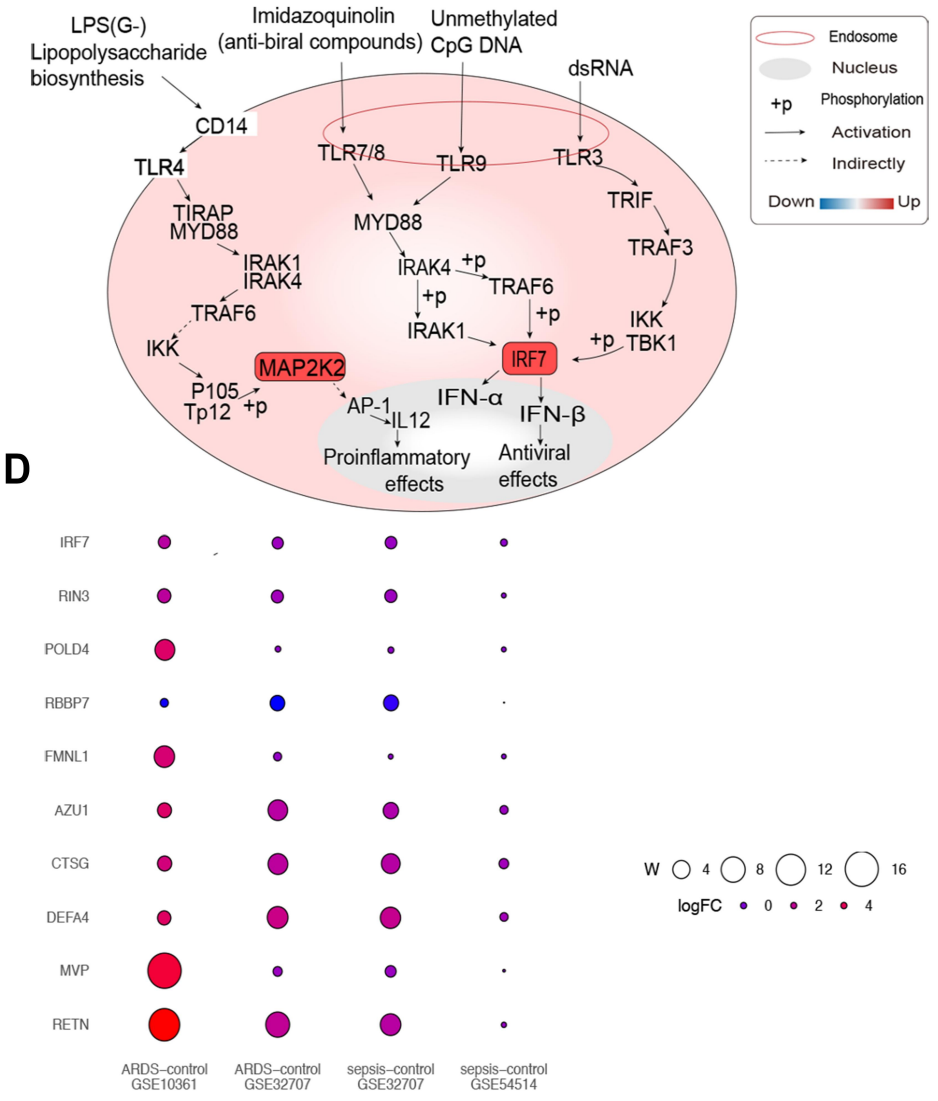

B

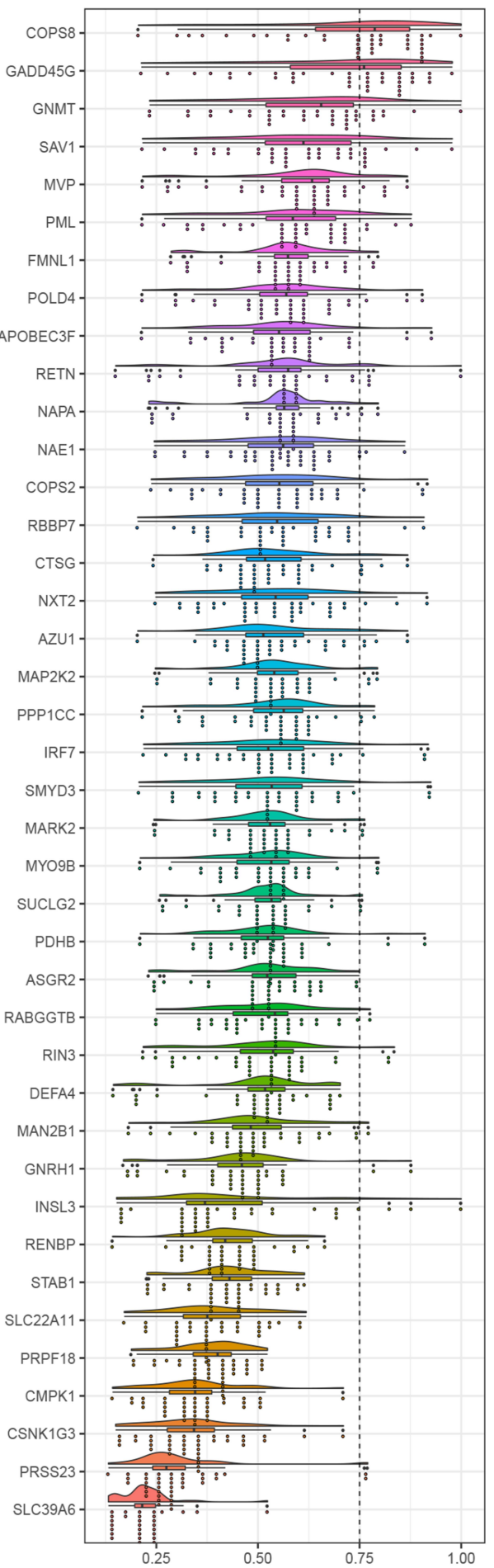

Figure 4 Protein-protein interaction network associated with hub genes. (A) Protein-protein interaction network of genes related to sepsis-induced ARDS. Red nodes represent up-regulated genes and blue nodes represent down-regulated genes. The red outer ring represents hypermethylation, and the blue outer ring represents hypomethylation. Brown triangles represent the pathway involved by genes (B) The potential mechanism of regulatory network pathways affecting genes related to sepsisinduced ARDS. Red squares represent up-regulated genes. (C) Average functional similarity among the $4 \mathrm{I}$ shared up-regulated and down-regulated genes related to sepsisinduced ARDS. (D) Bubble heat map of hub genes. $\mathrm{W}=$ mean functional similarity $\times$ mean $\mathrm{Pi}$. W represents the bubble size, and the logFc value is represented by different colors. 


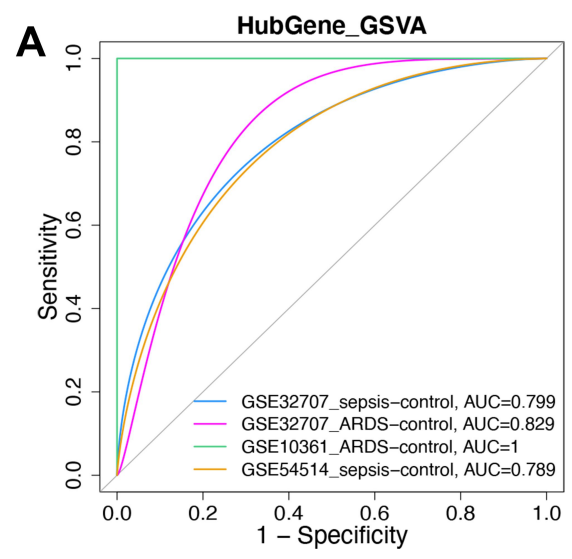

B
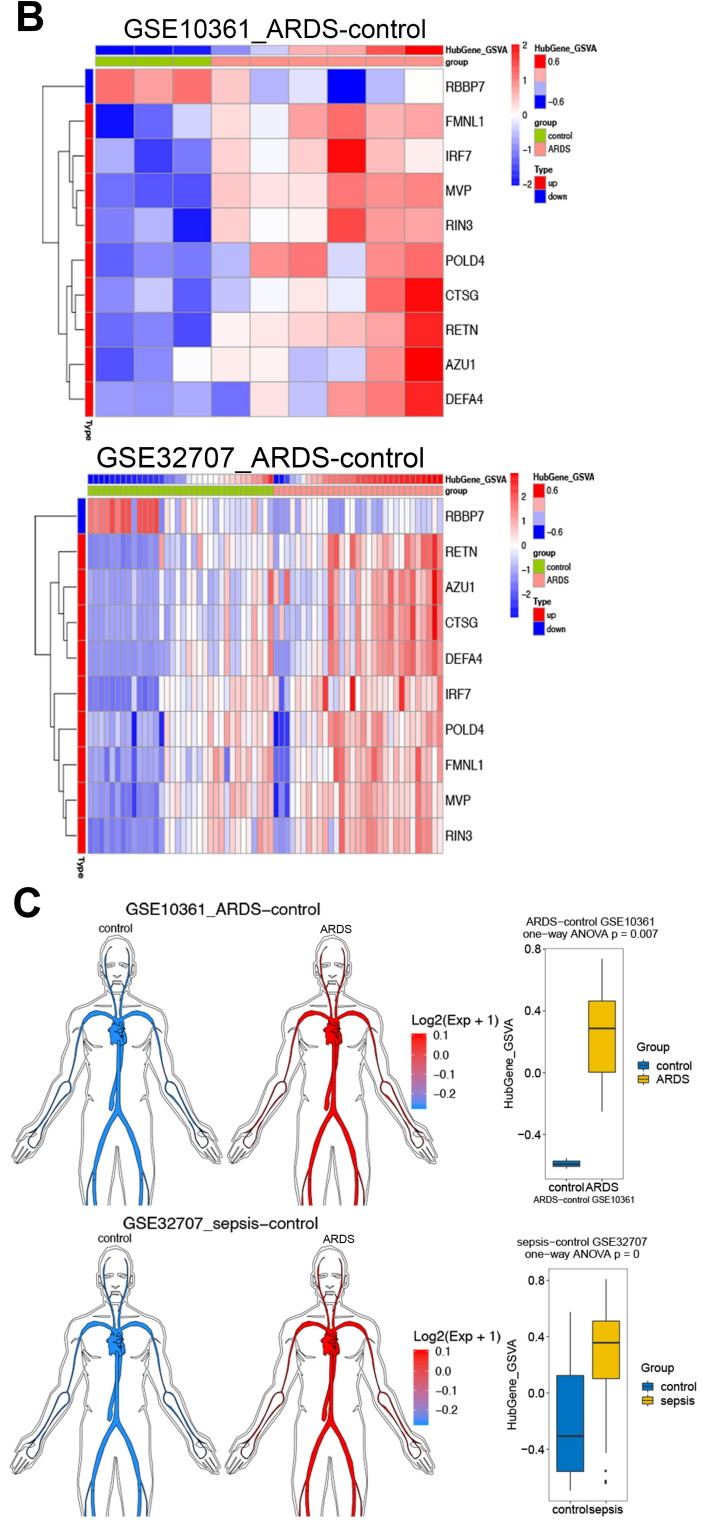

D
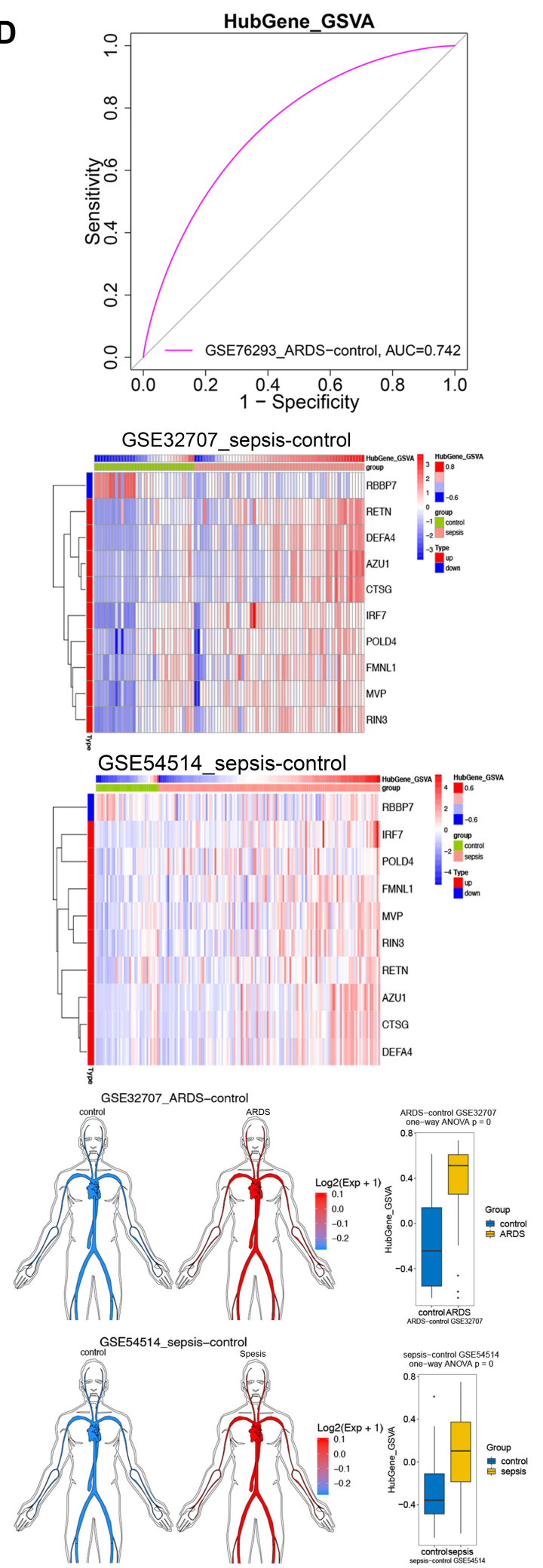

Figure 5 Expression of hub genes in sepsis-induced ARDS and their ability to predict the condition. (A) Receiver operating characteristic (ROC) curves showing the diagnostic potential of gene set variation analysis (GSVA) scores for different sets of hub genes. (B) Heatmap depicting hub gene expression and GSVA scores. (C) The hub gene GSVA score was higher in patients with sepsis or ARDS than in controls. (D) ROC curve analysis of hub gene GSVA in dataset GSE76293. 
A

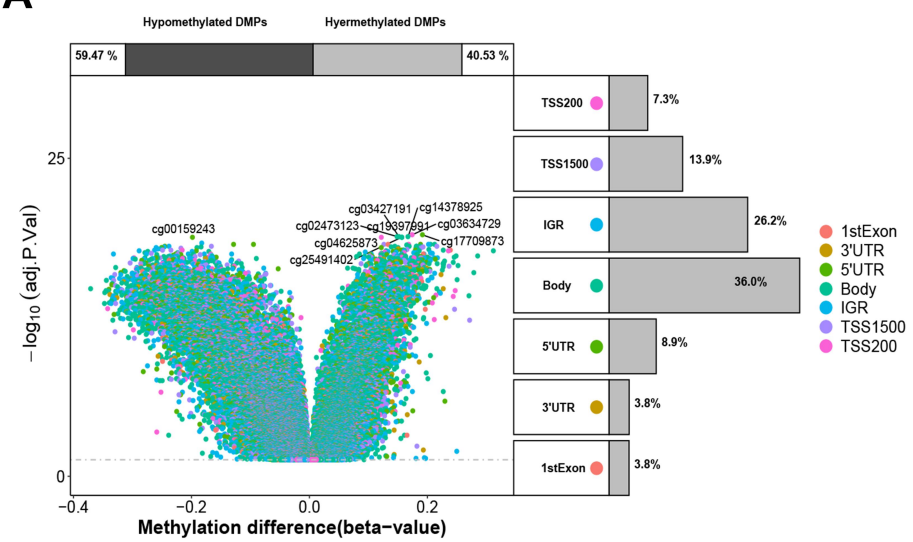

C

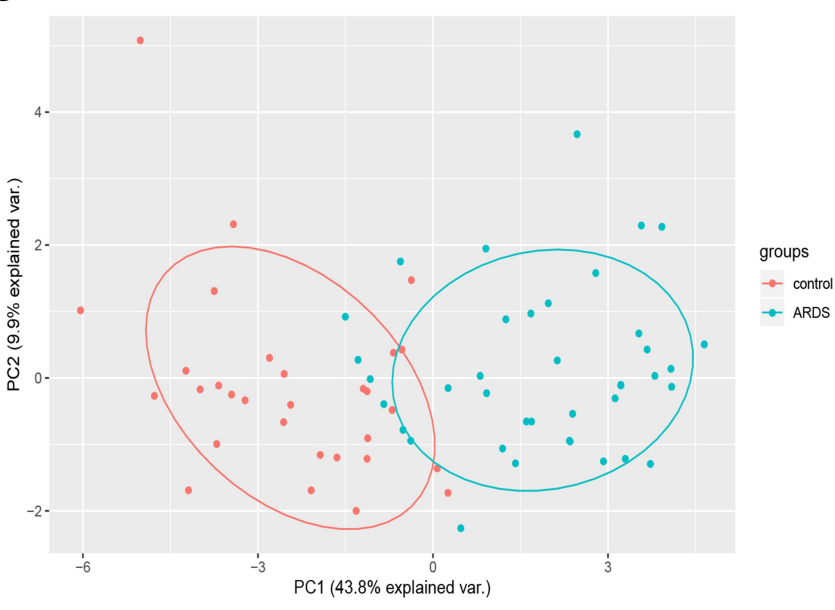

B
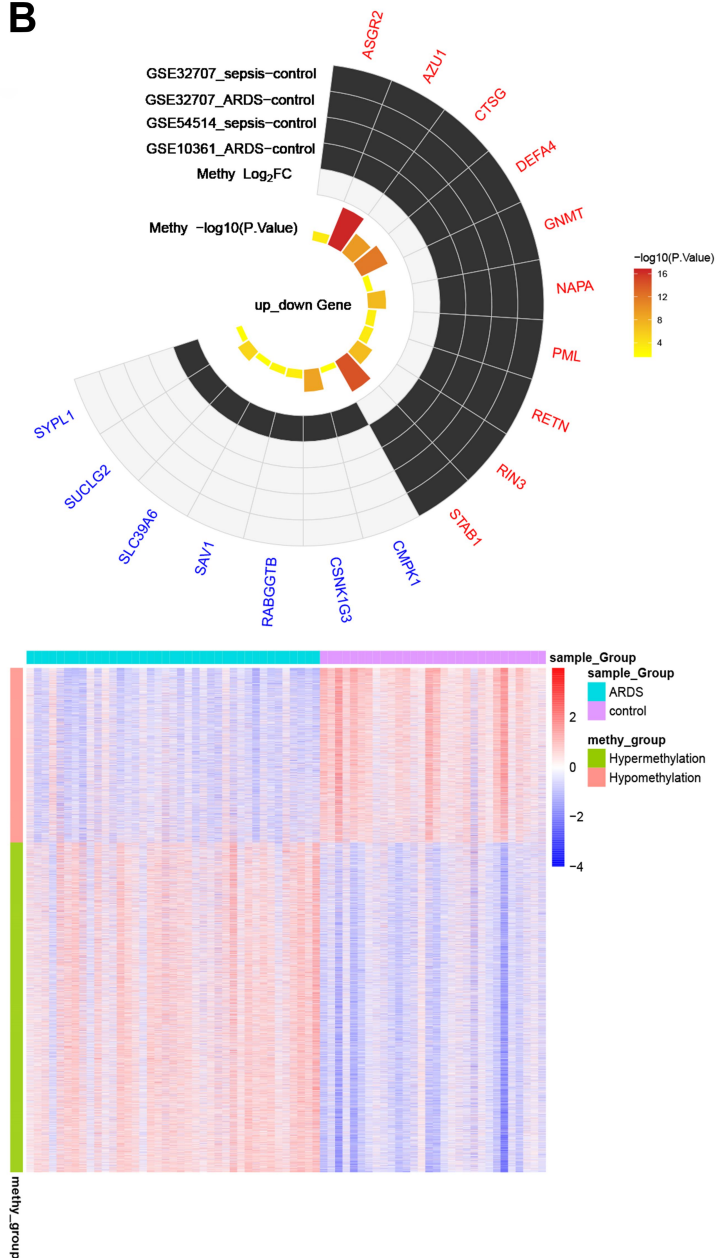

Figure 6 Differential methylation of hub genes. (A) Volcano plot of top differentially methylated genes in relation to the gene. Percentages of hyper- and hypomethylated genes are displayed on top, while the proportions of different genomic features are shown on the right. (B) The 17 differentially methylated genes related to sepsis-induced ARDS. Red represents up-regulated genes, and blue represents down-regulated genes. (C) Principal component analysis using the 17 differentially methylated genes. (D) Heatmap showing methylation levels of the 17 differentially methylated genes.

Abbreviations: IGR, intergenic region; TSS, transcription start site; UTR, untranslated region.

Our results should be interpreted with caution in light of several limitations. First, this study was a retrospective analysis of incomplete public datasets. Since we do not know the patient's infection level, treatment methods, or additional clinically relevant data, we cannot rule out that these factors may have confounded our analysis. Second, our study analyzed only three datasets for gene expression, one dataset for methylation, and one dataset for validation. Our results should be verified and extended with larger samples. Future work should also extend our bioinformatics analyses in the laboratory with tissue samples and cell cultures.

Despite these limitations, we have identified key genes showing aberrant expression in sepsis-induced ARDS, which may be due in part to hypomethylation. These genes may be useful as biomarkers for early diagnosis of ARDS and for identifying septic patients at greater risk of developing ARDS.

\section{Acknowledgments}

This study was supported by the National Natural Science Foundation of China (81960343), the Guangxi Natural Science Foundation (2017GXNSFAA198249), the Scientific Research Fund of the Population and Family Planning Commission of Guangxi Zhuang Autonomous Region (S2017009) and the High-level Medical Expert Training Program of Guangxi “139” Plan Fund (G201903027).

\section{Disclosure}

The authors report no conflicts of interest in this work. 


\section{References}

1. Metwaly S, Cote A, Donnelly SJ, Banoei MM, Mourad AI, Winston BW. Evolution of ARDS biomarkers: will metabolomics be the answer? Am J Physiol Lung Cell Mol Physiol. 2018;315(4): L526-L534. doi:10.1152/ajplung.00074.2018

2. Xue M, Sun Z, Shao M, et al. Diagnostic and prognostic utility of tissue factor for severe sepsis and sepsis-induced acute lung injury. $J$ Transl Med. 2015;13:172. doi:10.1186/s12967-015-0518-9

3. Ware LB, Koyama T, Billheimer DD, et al. Prognostic and pathogenetic value of combining clinical and biochemical indices in patients with acute lung injury. Chest. 2010;137(2):288-296. doi:10.1378/chest.09-1484

4. Barnett N, Ware LB. Biomarkers in acute lung injury-marking forward progress. Crit Care Clin. 2011;27(3):661-683. doi:10.1016/j. ccc.2011.04.001

5. Li Y, Wang $\mathrm{C}, \mathrm{Wu} \mathrm{X}$, et al. IRF3 and IRF7 contribute to diesel exhaust particles-induced pulmonary inflammation by mediating mTORC1 activation and restraining autophagy in mice. Eur J Immunol. 2020;50(8):1142-1153. doi:10.1002/eji.201948415

6. Hu X, Deng J, Yu T, et al. ATF4 deficiency promotes intestinal inflammation in mice by reducing uptake of glutamine and expression of antimicrobial peptides. Gastroenterology. 2019;156 (4):1098-1111. doi:10.1053/j.gastro.2018.11.033

7. Banerjee A, Shukla S, Pandey AD, et al. RNA-Seq analysis of peripheral blood mononuclear cells reveals unique transcriptional signatures associated with disease progression in dengue patients. Transl Res. 2017;186:62-78 e9. doi:10.1016/j.trs1.2017.06.007

8. Ware LB, Koyama T, Zhao Z, et al. Biomarkers of lung epithelial injury and inflammation distinguish severe sepsis patients with acute respiratory distress syndrome. Crit Care. 2013;17(5):R253. doi:10.1186/cc13080

9. El Gazzar M, Yoza BK, Hu JY, Cousart SL, McCall CE. Epigenetic silencing of tumor necrosis factor alpha during endotoxin tolerance. J Biol Chem. 2007;282(37):26857-26864. doi:10.1074/jbc.M704584200

10. Vogel Ciernia A, LaSalle J. The landscape of DNA methylation amid a perfect storm of autism aetiologies. Nat Rev Neurosci. 2016;17 (7):411-423. doi:10.1038/nrn.2016.41

11. Koch A, Joosten SC, Feng Z, et al. Author correction: analysis of DNA methylation in cancer: location revisited. Nat Rev Clin Oncol. 2018;15(7):467. doi:10.1038/s41571-018-0028-9

12. Long H, Yin H, Wang L, Gershwin ME, Lu Q. The critical role of epigenetics in systemic lupus erythematosus and autoimmunity. J Autoimmun. 2016;74:118-138. doi:10.1016/j.jaut.2016.06.020

13. Bansal A, Pinney SE. DNA methylation and its role in the pathogenesis of diabetes. Pediatr Diabetes. 2017;18(3):167-177. doi:10.1111/pedi.12521

14. Yang IV, Lozupone CA, Schwartz DA. The environment, epigenome, and asthma. $J$ Allergy Clin Immunol. 2017;140(1):14-23. doi:10.1016/j.jaci.2017.05.011

15. Wang HM, Bodenstein M, Markstaller K. Overview of the pathology of three widely used animal models of acute lung injury. Eur Surg Res. 2008;40(4):305-316. doi:10.1159/000121471

16. Tatemichi M, Hata H, Tazawa H, Nakadate T. Lipopolysaccharide induces aberrant hypermethylation of Hic-1 in mouse embryonic fibroblasts lacking p53 gene. Anticancer Res. 2008;28(4B):2101-2108.

17. Angrisano T, Pero R, Peluso S, et al. LPS-induced IL-8 activation in human intestinal epithelial cells is accompanied by specific histone H3 acetylation and methylation changes. BMC Microbiol. 2010;10:172. doi:10.1186/1471-2180-10-172

18. Szilagyi KL, Liu C, Zhang X, et al. Epigenetic contribution of the myosin light chain kinase gene to the risk for acute respiratory distress syndrome. Transl Res. 2017;180:12-21. doi:10.1016/j. trs1.2016.07.020
19. Dolinay T, Kim YS, Howrylak J, et al. Inflammasome-regulated cytokines are critical mediators of acute lung injury. Am J Respir Crit Care Med. 2012;185(11):1225-1234. doi:10.1164/rccm.201201$0003 \mathrm{OC}$

20. Juss JK, House D, Amour A, et al. Acute respiratory distress syndrome neutrophils have a distinct phenotype and are resistant to phosphoinositide 3-kinase inhibition. Am J Respir Crit Care Med. 2016;194(8):961-973. doi:10.1164/rccm.201509-1818OC

21. Ritchie ME, Phipson B, Wu D, et al. limma powers differential expression analyses for RNA-sequencing and microarray studies. Nucleic Acids Res. 2015;43(7):e47. doi:10.1093/nar/gkv007

22. Zhang J, Luo Y, Wang X, et al. Global transcriptional regulation of STAT3- and MYC-mediated sepsis-induced ARDS. Ther Adv Respir Dis. 2019;13:1753466619879840. doi:10.1177/1753466619879840

23. Gevaert O. MethylMix: an $\mathrm{R}$ package for identifying DNA methylation-driven genes. Bioinformatics. 2015;31(11):1839-1841. doi:10.1093/bioinformatics/btv020

24. Hanzelmann S, Castelo R, Guinney J. GSVA: gene set variation analysis for microarray and RNA-seq data. BMC Bioinform. 2013;14:7. doi:10.1186/1471-2105-14-7

25. Robin X, Turck N, Hainard A, et al. pROC: an open-source package for $\mathrm{R}$ and $\mathrm{S}+$ to analyze and compare ROC curves. BMC Bioinform. 2011;12:77. doi:10.1186/1471-2105-12-77

26. Yu G, Wang LG, Han Y, He QY. clusterProfiler: an R package for comparing biological themes among gene clusters. OMICS. 2012;16 (5):284-287. doi:10.1089/omi.2011.0118

27. Szklarczyk D, Morris JH, Cook H, et al. The STRING database in 2017: quality-controlled protein-protein association networks, made broadly accessible. Nucleic Acids Res. 2017;45(D1):D362-D368. doi:10.1093/nar/gkw937

28. Shannon P, Markiel A, Ozier O, et al. Cytoscape: a software environment for integrated models of biomolecular interaction networks. Genome Res. 2003;13(11):2498-2504. doi:10.1101/gr.1239303

29. Han Y, Yu G, Sarioglu H, et al. Proteomic investigation of the interactome of FMNL1 in hematopoietic cells unveils a role in calcium-dependent membrane plasticity. $J$ Proteomics. 2013;78:72-82. doi:10.1016/j.jprot.2012.11.015

30. Zou C, Wang J, Huang X, Jian C, Zou D, Li X. Analysis of transcription factor- and ncRNA-mediated potential pathogenic gene modules in Alzheimer's disease. Aging (Albany NY). 2019;11 (16):6109-6119. doi:10.18632/aging.102169

31. Takeuchi O, Akira S. Pattern recognition receptors and inflammation. Cell. 2010;140(6):805-820. doi:10.1016/j.cell.2010.01.022

32. Kovach MA, Standiford TJ. Toll like receptors in diseases of the lung. Int Immunopharmacol. 2011;11(10):1399-1406. doi:10.1016/j. intimp.2011.05.013

33. Zhou S, Wang G, Zhang W. Effect of TLR4/MyD88 signaling pathway on sepsis-associated acute respiratory distress syndrome in rats, via regulation of macrophage activation and inflammatory response. Exp Ther Med. 2018;15(4):3376-3384. doi:10.3892/ etm.2018.5815

34. Huang C, Pan L, Lin F, Dai H, Fu R. Monoclonal antibody against Toll-like receptor 4 attenuates ventilator-induced lung injury in rats by inhibiting MyD88- and NF-kappaB-dependent signaling. Int $\mathrm{J} \mathrm{Mol}$ Med. 2017;39(3):693-700. doi:10.3892/ijmm.2017.2873

35. Kantarjian H, Issa JP, Rosenfeld CS, et al. Decitabine improves patient outcomes in myelodysplastic syndromes: results of a phase III randomized study. Cancer. 2006;106(8):1794-1803. doi:10.1002/ cncr. 21792

36. Issa JJ, Roboz G, Rizzieri D, et al. Safety and tolerability of guadecitabine (SGI-110) in patients with myelodysplastic syndrome and acute myeloid leukaemia: a multicentre, randomised, dose-escalation phase 1 study. Lancet Oncol. 2015;16(9):1099-1110. doi:10.1016/S1470-2045(15)00038-8 
37. Huang X, Kong G, Li Y, et al. Decitabine and 5-azacitidine both alleviate LPS induced ARDS through anti-inflammatory/antioxidant activity and protection of glycocalyx and inhibition of MAPK pathways in mice. Biomed Pharmacother. 2016;84:447-453. doi:10.1016/ j.biopha.2016.09.072

38. Lei C, Jiao Y, He B, Wang G, Wang Q, Wang J. RIP140 down-regulation alleviates acute lung injury via the inhibition of LPS-induced PPARgamma promoter methylation. Pulm Pharmacol Ther. 2016;37:57-64. doi:10.1016/j.pupt.2016.02.001
39. Wang X, Lei D, Ding J, et al. A DNA-methylated sight on autoimmune inflammation network across RA, pSS, and SLE. J Immunol Res. 2018;2018:4390789. doi:10.1155/2018/4390789

40. Francis M, Pandya M, Gopinathan G, et al. Histone methylation mechanisms modulate the inflammatory response of periodontal ligament progenitors. Stem Cells Dev. 2019;28(15):1015-1025. doi:10.1089/ scd.2019.0125

\section{Publish your work in this journal}

The International Journal of General Medicine is an international, peer-reviewed open-access journal that focuses on general and internal medicine, pathogenesis, epidemiology, diagnosis, monitoring and treatment protocols. The journal is characterized by the rapid reporting of reviews, original research and clinical studies across all disease areas. The manuscript management system is completely online and includes a very quick and fair peer-review system, which is all easy to use. Visit http://www.dovepress.com/ testimonials.php to read real quotes from published authors.

Submit your manuscript here: https://www.dovepress.com/international-journal-of-general-medicine-journal 